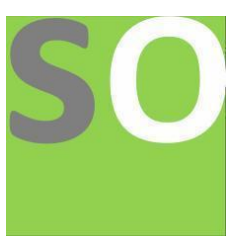

Article title: Three color theorem and Four color theorem

Authors: Zhaosheng Zhang[1]

Affiliations: Huaian Gaoliangjian Primary School, Hongze District, Huaian City, Jiangsu Province, China[1]

Orcid ids: 0000-0001-7465-0522[1]

Contact e-mail: ddr123@sina.com

License information: This work has been published open access under Creative Commons Attribution License $\mathrm{http}: / / c r e a t i v e c o m m o n s . o r g / l i c e n s e s / b y / 4.0 /$, which permits unrestricted use, distribution, and reproduction in any medium, provided the original work is properly cited. Conditions, terms of use and publishing policy can be found at https://www.scienceopen.com/.

Preprint statement: This article is a preprint and has not been peer-reviewed, under consideration and submitted to ScienceOpen Preprints for open peer review.

DOI: 10.14293/S2199-1006.1.SOR-.PP36ZX1.v1

Preprint first posted online: 21 February 2022

Keywords: Plane graph, Coloring, Four color theorem,Three color theorem 


\title{
Three color theorem and Four color theorem
}

\author{
ZhaoshengZhang
}

Huaian Gaoliangjian Primary School, Hongze District, Huaian City, Jiangsu Province, China

Email: ddr123@sina.com

\begin{abstract}
According to the arrangement order of the vertexes in the line, the two-color theorem is derived. Add auxiliary lines to the vertexes that do not conform to the four-color closed chain, the one-color theorem and the two-color theorem, the coloring order of each vertex is locked, and the three-color theorem is derived. According to the one-color theorem, the two-color theorem, the three-color theorem and the law of chain structure, the four-color theorem is derived.
\end{abstract}

AMS 2010 SUBJECT CLASSIFICATIONS:Primary 05-XX, 05Cxx; secondary $05 C 15$.

KEYWORDS AND PHRASES: Plane graph, Coloring, Four color theorem, Three color theorem

\section{Introduction}

A student named Francis Guthrie at British University proposed the four-color conjecture in 1852 , the four-color problem has not been solved so far, this article gives the solution to the four-color problem.

\section{Four color theorem}

Four-color problem: Any map is drawn with $\mathrm{N}(\mathrm{N}$ is a natural number, $1 \leqslant \mathrm{~N} \leqslant 4)$ colors, colouring countries with common borders differently. (Excluding enclaves)

A country on the map is used as a vertex, if two countries have a common border, the two vertexes are connected to form a line segment, this line segment is 1 side. The four colors are color-1, color-2, color-3, and color-4, Denoted as 1, 2, 3, and 4 in the figure.

\subsection{One color theorem}

One-color theorem: The vertexes in the plane graph are not connected, the vertexes in the plane graph are colored with 1 color.

\subsection{Two color theorem}

Proof: There are $\mathrm{N}(\mathrm{N}$ is a natural number, $\mathrm{N} \geqslant 1)$ closed lines (there are $\mathrm{W}$ vertexes in each closed line, $\mathrm{W}$ is an even number, $\mathrm{W} \geqslant 4$ ) in the plane graph, these closed lines have 
shared vertexes (Figure 1). Any two closed lines (each closed line has $M$ vertexes, $M$ is an even number, $M \geqslant 4$ ) in the plane graph, when two closed lines share an even number of vertexes, each closed line has an even number of unshared vertexes; When two closed lines share an odd number of vertexes, each closed line has an odd number of unshared vertexes. Similarly, the vertexes in the plane graph are colored with 2 colors, the order of the colors of the vertexes in each closed line is not affected by the shared vertexes. thereby verifying the two-color theorem.

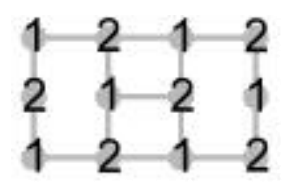

Figure. 1. Two color structure

\subsection{Chain structure}

One closed line (there are 3 vertexes in the closed line) has an odd number of vertexes, the 3 vertexes in this closed line must be colored with 3 colors. In the plane graph, the area outside of a closed line (there are 3 vertexes in the closed line) is divided into 2 areas by the closed line, one area does not affect the vertex coloring in the other area, it is equivalent to one area can ignore the other area.

1-level chain structure: $\mathrm{N}(\mathrm{N}$ is a natural number, $\mathrm{N} \geqslant 2)$ closed lines (there are 3 vertexes in the closed line) form a chain structure, each closed line shares $M(M$ is a natural number, $1 \leqslant$ $M \leqslant 2$ ) vertexes with another closed line. The vertexes in the plane graph are colored with 3 colors, the closed line a $\beta \gamma$ and the closed line $\beta \gamma \delta$ share vertexes $\beta$ and $\gamma$ (Left 1 in figure 2). Vertexes $\alpha$ and $\delta$ are locked and must be colored in the same color, these 2 vertexes are in the same color vertex position of this chain. Vertexes $\varepsilon$ and $\zeta$ are locked with 2 colors, the order of the vertex $\beta$ and $\gamma$ coloring color is not locked. There is 1-layer of vertexes in the same color vertex position in a chain (Left 1, left 2, left 3, right 1 , and right 2 in figure 2).

1 chain: There is a layer of vertexes in the same color vertex position in a chain (First left, second left, third left, third right, and second right in figure 2), one chain can have branches.

Three-color closed chain: The vertexes in the same color vertex position in a closed chain are not connected, the vertexes in this closed chain are colored with 3 colors (Right 3 in figure 2).

Four-color closed chain: The vertexes in the same color vertex position in a closed chain are connected, the vertexes in this closed chain are colored with 4 colors (Left 2 and right 2 in figure 2).

1 vertex in a closed chain is colored with color-4, E.g vertex $\varepsilon$ (Right 2 in figure 2), and the uncolored vertexes no longer constitute closed chain. The vertexes in a chain structure are colored with 3 colors, This chain structure has $\mathrm{N}(\mathrm{N}$ is a natural number, $\mathrm{N} \geqslant 3)$ closed lines (3 vertexes in each closed line), and each closed line shares 2 vertexes with another closed line, the order of the colors of each vertex in this chain structure is locked (Right 1 in figure 2). The $\mathrm{M}-$ level $(\mathrm{M}$ is a natural number, $\mathrm{M} \geqslant 2$ ) chain structure is similarly. 


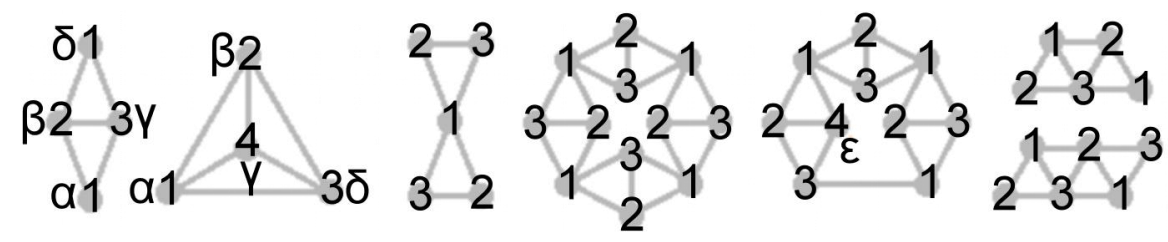

Figure. 2. 1-level chain structure

A chain structure: The vertexes in the plane graph are colored with 3 colors, the order of the colors of each vertex is locked, and all of these vertexes are in the same chain structure. The vertexes of the same color in a chain are the vertexes of the order of locked colors in a chain structure, and this chain is in this chain structure.

$\mathrm{N}$-level ( $\mathrm{N}$ is a natural number, $\mathrm{N} \geqslant 1$ ) chain structure: Vertex $a$ connects vertex $\theta$ and 1 to form a 2-level chain structure, vertex $\theta$ and $\quad l$ are in the same 1-level chain structure, the order of the colors of vertex $\theta$ and $l$ is locked, the colors of vertex $\theta$ and $\quad l$ are different. Vertex $\zeta$ connects vertex $\alpha$ and $\beta$ to form a 3-level chain structure, vertex $\alpha$ and $\beta$ are in the same 2-level chain structure, the order of the colors of vertex $\alpha$ and $\beta$ is locked, the colors of vertex $\alpha$ and $\beta$ are different. Vertex $\theta$ and $l$ are the first-level vertexes in the chain structure, vertex a, $\beta, \gamma, \delta$, and $\varepsilon$ are the 2 -level vertexes in the chain structure, vertex $\zeta$ and $\eta$ are the 3-level vertexes in the chain structure (Figure 3). The chain structure has $\mathrm{N}-l e v e l(\mathrm{~N}$ is a natural number, $\mathrm{N} \geqslant 1)$. 1 vertex in a $\mathrm{N}$-level $(\mathrm{N}$ is a natural number, $N \geqslant 1$ ) closed chain is colored with color-4, E.g vertexes $\eta$ (Figure 3), the Uncolored vertexes no longer form a closed chain.

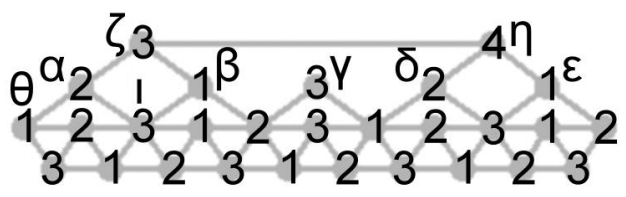

Figure. 3. Multi-level chain structure

\subsection{Three color theorem}

The three-color conjecture: There are no four color closed chains in the closed chain, the vertexes in the plane graph colored with 3 colors, making the colors of the connected vertexes different.

Proof: The two endpoints of the line segment a $\beta$ are arbitrary colors, 1 vertex in the line segment a $\beta$ is colored with color-3, and the other vertexes can definitely be colored with color-1, color-2. The vertexes in the line segment a $\beta$ colored with 3 colors (Left 1 in figure 4).

Three-color structure: Each vertex in the three-color structure is not in the chain structure, each vertex is connected with $\mathrm{N}(\mathrm{N}$ is a natural number, $\mathrm{N} \geqslant 3)$ vertexes, and each vertex does not conform to the one-color theorem and two-color theorem (Left 2 in figure 4).

Method of adding auxiliary lines: Adding auxiliary lines to vertexes $\delta, \varepsilon$, and $\zeta$, each auxiliary vertex in the auxiliary line $\delta \zeta$ connects the vertex $\varepsilon$ to form a chain structure. When vertexes $\delta$ and $\zeta$ have the same color, there are $\mathrm{N}(\mathrm{N}$ is an odd number) auxiliary 
vertexes in the auxiliary line. When vertexes $\delta$ and $\zeta$ have different colors, there are M (M is an even number) auxiliary vertexes in the auxiliary line. The vertexes in the chain structure can colored with 3 colors (Right 1 and right 2 in figure 4).
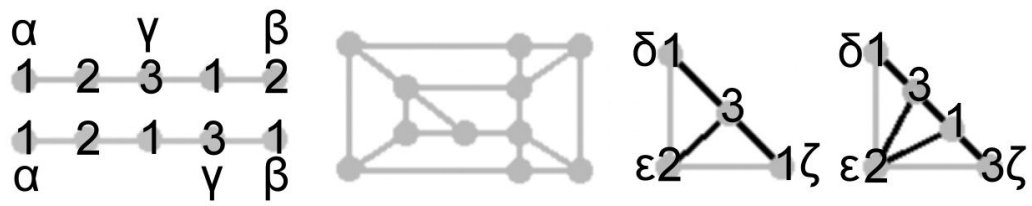

Figure. 4. Three-color structure

The vertexes $\alpha$ and $\beta$ of vertex position of the same color in a four-color closed chain and 1 vertex $\delta$ in the three-color structure, Add auxiliary lines to these 3 vertexes, Add a new four-color closed chain (Left 1 and left 2 in figure 5). The vertexes $\alpha, \beta$, and $\gamma$ of vertex position of the same color in a four-color closed chain, vertex a connects to vertex $\gamma$. The vertex $\delta$ in the three-color structure connects the vertexes a and $\beta$, add auxiliary lines to the vertexes $\delta, \alpha$, and $\beta$, there are $\mathrm{N}(\mathrm{N}$ is a natural number) auxiliary vertexes in the auxiliary line, Add a new four-color closed chain. When $\mathrm{N}$ is an odd number, the original four-color closed chain and the new four-color closed chain share the vertexes $a$ and $\gamma$ of vertex position of the same color (Left 1 in figure 5). When $\mathrm{N}$ is an even number, The two endpoints of a new edge are the vertexes of vertex position of the same color in the new four-color closed chain, E.g the new edge a $\varepsilon$ (Left 2 in figure 5).

The two vertexes are not at the same time are the vertexes of vertex position of the same color in the same four-color closed chain, add auxiliary lines to these 2 vertexes and 1 vertex in the three-color structure, it can be done without adding new four-color closed chains, E.g (Right 1 and right 2 in figure 5).

$\mathrm{N}(\mathrm{N}$ is a natural number, $\mathrm{N} \geqslant 2)$ level chain structure is similarly.

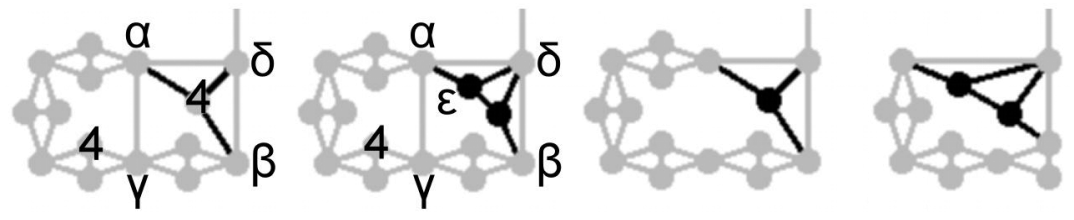

Figure. 5. Four-color structure plus auxiliary line

There are no four-color closed chain in the plane graph. Add auxiliary lines to each vertex in the three-color structure and the two endpoints of the side connecting the two chain structures, make each connected vertexes in the three-color structure and the chain structure be in the same chain structure. After adding auxiliary lines to the vertexes in this plane graph, it can be done without adding new four-color closed chains. Color with 3 colors, the order of the colored colors of each vertex in each chain structure is locked (Figure 6). The vertexes in this plan can be colored in 3 colors, the three-color theorem holds. 


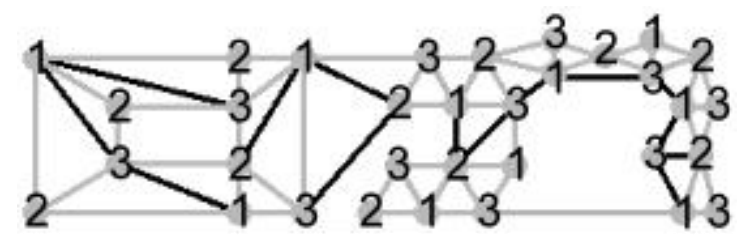

Figure. 6. Three color auxiliary line

\subsection{Four color theorem}

Four-color conjecture: There are four color closed chains in the plane graph, the vertexes in the plane graph can definitely be color in 4 colors, making the colors of the connected vertexes different.

Proof: The vertexes in the plane graph are not colored, use color-4 to color the $\mathrm{N}(\mathrm{N}$ is a natural number, $\mathrm{N} \geqslant 1$ ) vertexes in each closed chain, the uncolored vertexes in each closed chain no longer form closed chain. The area other than 1 closed chain in the plane graph is divided into 2 area by this closed chain, the vertexes in $M$ ( $M$ is a natural number, $1 \leqslant M \leqslant 2$ ) area are uncolored, the vertexes in this closed chain colored first, then color the vertexes in the area where the vertexes are uncolored. In this article, it is set up that the area where the vertexes are not colored is the inner area, the area where the vertexes are colored is the outer area.

A 1-level closed chain has 2 layers of vertexes, the vertexes of the outer layer form a closed line. The vertexes in this closed chain are not colored, It can definitely be colored with colors 4 the $\mathrm{N}(\mathrm{N}$ is a natural number, $\mathrm{N} \geqslant 1$ ) vertexes of the inner layer of this closed chain, E.g. vertex $\varepsilon$ (Right 2 in figure 2).

In this article, the connection amount of the vertexes in each structure takes the maximum value. When the connection amount of the vertexes in a structure takes the maximum value, can make up every form of this structure.

Zone: A structure of composed $\mathrm{N}(\mathrm{N}$ is a natural number, $\mathrm{N} \geqslant 2$ ) layer vertexes (the connection amount of the vertexes takes the maximum value).

Multiple closed chains in the plane graph can share 1 vertex. There are $\mathrm{N}(\mathrm{N}$ is a natural number, $N \geqslant 4$ ) vertexes in a closed zone (there are 2 layers of vertexes in the closed zone). The vertex $\alpha$ is at position of the same color vertex of a chain. Starting from the vertexes $\alpha$, The 1-level chain a $\gamma \delta$ is formed in the clockwise direction, and the 1-level chain a $\varepsilon \beta$ is formed in the counterclockwise direction, there are $M(M$ is a natural number, $0 \leqslant M \leqslant 2 N)$ 1-level four-color closed chain in this closed zone (Figure 8). The inner layer of this closed zone has $\mathrm{X}(\mathrm{X}$ is a natural number, $\mathrm{X} \geqslant 1)$ vertexes. When $\mathrm{X}$ is an even number greater than zero, the inner layer has $\mathrm{Y}(\mathrm{Y}$ is a natural number, $2 \leqslant \mathrm{Y} \leqslant \mathrm{X} / 2)$ vertexes that can be colored with color-4; When $\mathrm{X}$ is an odd number greater than 1, the inner layer has $\mathrm{Y}(\mathrm{Y}$ is a natural number, $2 \leqslant Y \leqslant(X-1) / 2)$ vertexes that can be colored with color-4. The area other than 1 closed zone in the plane graph is divided into 2 area by this closed zone, the vertexes in $\mathrm{Z}$ ( $\mathrm{Z}$ is a natural number, $1 \leqslant Z \leqslant 2$ ) area are uncolored, the vertexes in this closed zone (there are 2 layers of vertexes in the closed zone) colored first, then color the vertexes in the area where the vertexes are uncolored.

1 closed zone (there are 2-layers of vertexes in the closed zone) have $\mathrm{N}(\mathrm{N}$ is a natural number, $1 \leqslant N \leqslant 3)$ vertexes in the inner layer, and $M(M$ is a natural number, $M \geqslant 4)$ vertexes 
in the outer layer. $X(X$ is a natural number, $1 \leqslant X \leqslant 3$ ) vertexes of the inner layer of this closed zone connects to the $\mathrm{Y}$ ( $\mathrm{Y}$ is a natural number, $\mathrm{Y} \geqslant 3$ ) vertexes of the outer layer, E.g vertexes $\alpha$ and $\varepsilon$ (Figure 7). In this closed zone, Edge $\alpha \beta$ or closed line $\gamma \delta \varepsilon$ is in a closed chain, vertex $a$ or vertex $\varepsilon$ are also in this closed chain. So 1 vertex in this closed zone can be colored with color-4, this vertex connects the $\mathrm{Y}(\mathrm{Y}$ is a natural number, $\mathrm{Y} \geqslant 3$ ) vertex of the outer layer, the uncolored vertexes in this closed zone no longer form closed chain.

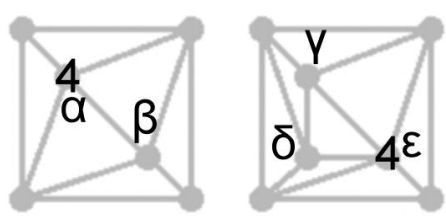

Figure. 7. 2-layer vertexes structure

In this article, except the closed zone of clearly illustrate, other closed zone (there are 2-layers of vertexes in the closed zone) have $\mathrm{N}(\mathrm{N}$ is a natural number, $\mathrm{N} \geqslant 4)$ vertexes in the inner layer, and $\mathrm{M}(\mathrm{M}$ is a natural number, $\mathrm{M} \geqslant 4)$ vertexes in the outer layer.

Assuming that vertex $\zeta$ is connected to vertex 1 . Vertex $\zeta, \theta$, and 1 form a closed line, therefore, vertexes $\zeta$ and 1 cannot be connected. The inner layer of this closed zone have $\mathrm{N}$ ( $\mathrm{N}$ is a natural number, $\mathrm{N} \geqslant 2$ ) vertexes, the vertexes of these inner layer are not connected to each other, and each vertex is connected to $M$ ( $M$ is a natural number, $M \geqslant 2$ ) vertexes of the outer layer, E.g vertexes $\beta, \delta, \eta$ and 1 (Left 1 and left 2 in figure 8).

The vertex $\kappa$ of the inner layer of 1 closed zone (there are 2-layers of vertexes in the closed zone) connects to 1 vertex of the outer layer. Assuming vertex $\kappa$ is colored color- 4 , the uncolored vertexes form 21 -level closed chains. There must be $\mathrm{M}$ ( $\mathrm{M}$ is a natural number, $\mathrm{M} \geqslant$ 2) inner layer vertexes can be colored color- 4 , but only $M(M$ is a natural number, $M \geqslant 1)$ inner layer vertexes can be colored color-4. So vertex $\kappa$ sometimes cannot be colored with color-4. (Left 3 in figure 8).

Structure I: The inner layer vertex $\mu$ of a closed zone (there are 2-layers of vertexes in the closed zone) connects the 2 vertexes of outer layer. Assuming that the vertex $\mu$ is colored with color -4 , the uncolored vertexes constitute $\mathrm{N}(\mathrm{N}$ is a natural number, $\mathrm{N} \leqslant 1)$ closed chains. There are $M(M$ is a natural number, $M \geqslant 1)$ uncolored vertexes in the inner layer, these vertexes are not connected to the vertex $\mu$, and not connected to each other, and connect the $\mathrm{X}$ ( $X$ is a natural number, $X \geqslant 2$ ) vertexes of the outer layer, and can be colored with color-4; if the vertexes of except vertex $\mu$ in this closed zone form closed chain, these vertexes in this closed chain, E.g. vertex $\xi$; these vertexes and vertex $\mu$ are colored with color-4, uncolored vertexes no longer constitute closed chain (Right 2 in figure 8).

Structure II: The vertexes 0 in the inner layer of a closed zone (there are 2 layers of vertexes in the closed zone) connect the $\mathrm{N}(\mathrm{N}$ is a natural number, $\mathrm{N} \geqslant 3$ ) vertexes of the outer layer, vertexes $\mathrm{o}$ is colored with color- 4 , uncolored vertexes no longer constitute a closed chain (Right 1 in figure 8). 

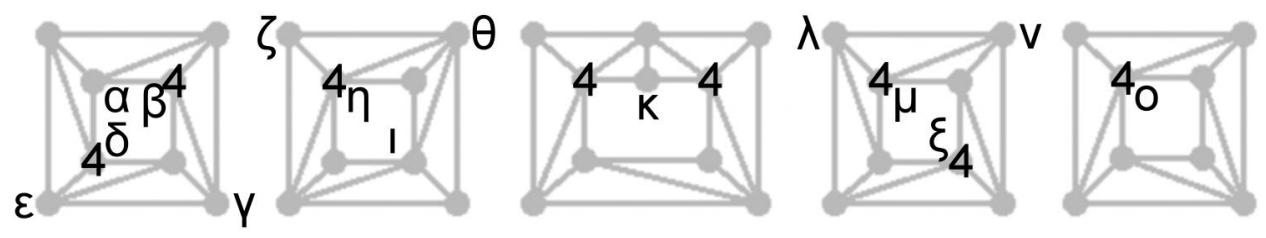

Figure. 8. Closed zone

Add 1 new connection: Add 1 connection in 2 vertexes of the inner layer of a closed zone (there are 2 layers of vertexes in the closed zone), newly add $\mathrm{N}(\mathrm{N}$ is a natural number, $\mathrm{N} \leqslant 2$ ) closed chains and 1 side. The newly added closed chain shares the new side and 2 vertexes in the new side, E.g vertex $\gamma$ connects to vertex $\delta$ (Left 2 in figure 9).

Structure III: 1 closed zone (there are 2 layers of vertexes in the closed zone) 2 vertexes in the inner layer are not connected to each other, and each connects to the 2 vertexes of the outer layer, add 1 new connection to these 2 vertexes. When the vertexes of the inner layer are connected to $\mathrm{N}(\mathrm{N}$ is a natural number, $1 \leqslant \mathrm{~N} \leqslant 2)$ vertexes of the outer layer, in the inner layer vertexes that do not increase the connection, there are $M(M$ is a natural number, $M \geqslant 2)$ vertexes that are not connected to each other, and each are connected to the 2 vertexes of the outer layer, these vertexes in the inner layer can be colored with color-4, E.g the vertexes a and $\beta$ (left 1 in Figure 9), uncolored vertexes no longer form closed chain. $X$ ( $X$ is a natural number, $X \geqslant 1$ ) vertexes in the inner layer are connected to $Y(Y$ is a natural number, $Y \geqslant 3$ ) vertexes in the outer layer, in the inner layer vertexes that do not increase the connection, there are $X(X$ is a natural number, $X \geqslant 1)$ vertexes that are not connected to each other, and each are connected to the $\mathrm{Y}(\mathrm{Y}$ is a natural number, $\mathrm{Y} \geqslant 3$ ) vertexes of the outer layer, these vertexes can be colored with color-4, E.g the vertex $\varepsilon$ (Left 3 in figure 9), uncolored vertexes no longer form closed chain. If the vertexes of the inner layer that can be colored with color-4 are on the same side of the new edge, the uncolored vertexes on the same side no longer form closed chain, the vertexes on the same side and new edges form a new four-color closed chain at most; The vertex on the other side of the new edge and the new edge form a new four-color closed chain at most, colored with color-4 one vertex in the inner layer of this closed chain, E.g the vertex $\zeta$ (Left 3 in Figure 9), uncolored vertexes no longer form closed chain.

There are 4 vertexes in the inner layer of a closed zone, The 2 vertexes of the inner layer are add 1 new connection, the 4 vertexes in the inner layer form a chain structure, E.g the vertexes $\alpha, \beta, \mu$, and $v$ constitute a chain structure ( Left 1, left 2 and left 3 in figure 9). Edge $\mu \vee$, closed line $\mu \vee \alpha$ and closed line $\mu \vee \beta$ are in a closed chain, the vertexes $a$ and $\beta$ are also in this closed chain. The vertexes $\alpha$ and $\beta$ and vertexes $\varepsilon$ and $\zeta$ are vertexes of the same color in the new chain structure, and they are all colored with color- 4 , the uncolored vertexes in the new closed chain no longer form the closed chain.

Structure IV: The 2 vertexes of the inner layers of a closed zone (there are 2 layers of vertexes in the closed zone) increase 1 new connection. Among the two vertexes, there are $\mathrm{N}(\mathrm{N}$ is a natural number, $\mathrm{N} \leqslant 2$ ) vertexes each connects to the $\mathrm{M}(\mathrm{M}$ is a natural number, $\mathrm{M} \geqslant 3$ ) vertexes of the outer layer. One of these two vertexes can definitely be be colored with color-4. This vertex is connected to the outer layer $X(X$ is a natural number, $X \geqslant 3$ ) vertexes, E.g vertex $\gamma$ (Second left in figure 9), uncolored vertexes no longer constitute a closed chain.

Add $\mathrm{N}(\mathrm{N}$ is a natural number, $\mathrm{N} \geqslant 1$ ) new connections: 
Structure V: Vertexes $\eta$ and $l$ of the inner layer of Structure III are colored with color-4, the vertex $\eta$ and the vertex $\quad l$ are the vertexes that do not increase the connection and each connects the 2 vertexes of the outer layer. The vertex $\quad l$ and the vertexes of the inner layer add 1 new connections, add $\mathrm{M}(\mathrm{M}$ is a natural number, $\mathrm{M} \geqslant 1)$ new closed zone. Vertex 1 connects $X(X$ is a natural number, $X \geqslant 3)$ outer vertexes, In the new closed zone, vertex 1 connects the $X(X$ is a natural number, $X \geqslant 3)$ vertexes of the outer layer, constitute structure II , E.g the vertex 1 of the inner layer of the new closed zone connects the vertexes $0, \pi$, and $\kappa$ of the outer layer (Right 1 in figure 9). If vertex $\pi$ is connected to vertex $\xi$, the vertexes $1, \pi$, and $\xi$ form a closed line, vertex 1 connects 2 vertexes of the outer layer of a new closed zone(Right 2 in figure 9), uncolored vertexes no longer constitute a closed chain.

in the plane graph, in each closed zone (there are 2 layers of vertexes in the closed zone) definitely have one of the V structures. Similarly, first use color-4 to color the vertexes in each closed zone, uncolored vertexes no longer constitute a closed chain.
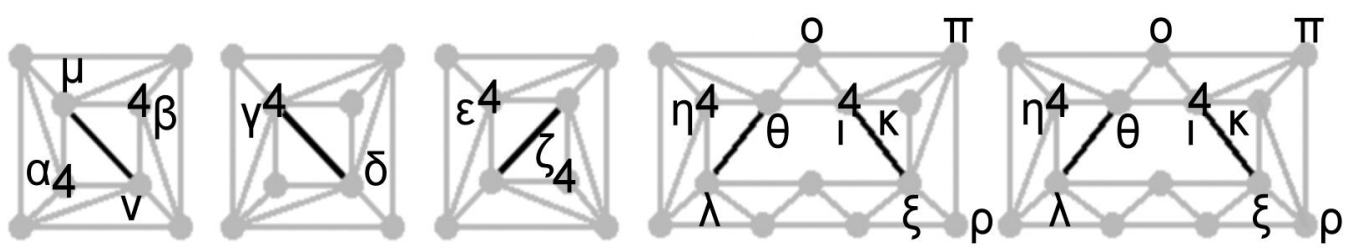

Figure. 9. Increase the connection of inner layer vertexes

A N-level ( $\mathrm{N}$ is a natural number, $\mathrm{N} \geqslant 2$ ) closed chain have $\mathrm{M}$-layer ( $\mathrm{M}$ is a natural number, $M \geqslant 3$ ) vertexes (Figure 3 ), the $X(X$ is a natural number, $X \geqslant 1$ ) vertexes in the inner layer of this closed chain can definitely be be colored with color-4.

After the vertexes in the four-color closed chain in the plane graph are colored with one color, according to the one-color theorem, the two-color theorem and the three-color theorem. The uncolored vertexes no longer constitute the four-color closed chain and can definitely be be colored in the other 3 colors. The vertexes in the plane graph can definitely be color in 4 colors, thereby verifying the four-color theorem.

\section{References}

[1] Saaty TL, Kainen PC The Four-colour. Problem-Assauts and Conquest London: Ma Graw-Hill Inc, 1977.

[2] OreO, The Four Colour Problem, New York: Acdemic Press, 1976.

[3] John A. Koch, Rudolf Fritsch, Gerda Fritsch, and J. Peschke, The Four-Color Theorem, The American Mathematical Monthly 106(8):785, October 1999, DOI:10.2307/2589042

[4] Gary Chartrand, Stephen T. Hedetniemi, Futaba Okamoto, and Ping Zhang, A four colorings theorem, Journal of Combinatorial Mathematics and Combinatorial Computing 77, May 2011, ISBN: 0835-3026

[5] Gary Chartrand and Ping Zhang, Chromatic Graph Theory (pp.461-474), November 2019, DOI:10.1201/9780429438868-18 
[6] C. Thomassen, Grötzsch' s 3-Color Theorem and Its Counterparts for the Torus and the Projective Plane, Journal of Combinatorial Theory, Series B, Volume: 62, Pages: 268-279, November 1994, DOI: $10.1006 /$ jctb. 1994.1069

[7] V. Vilfred, The Four Color Theorem - A New Proof by Induction, 2017-01-11, ArXiV ID: 1701.03511

[8] Rudolf Fritsch, Gerda Fritsch, The Four-Color Theorem, 1998, DOI: 10.1007/978-1-4612-1720-6

[9] Neil Robertson, Daniel P. Sanders, Paul Seymour, Robin Thomas, Reducibility in the Four-Color Theorem, 24 January 2014, ArXiV ID: 1401.6481

[10] Wei-Chang Yeh, A Simple Proof for the Four-Color Theorem, 01 May 2019, ArXiV ID: 1905.00255

[11] X. -J. Wang, T. -Q. Wang, A Study on Hand Proof for The Four-Color Theorem, 27 April 2021, ArXiV ID: 2104.14399

[12] André Luiz Barbosa, A Human-Checkable Four-Color Theorem Proof, 06 June 2017, ArXiV ID: 1708.07442

[13] Neil Robertson, Daniel P. Sanders, Paul Seymour, Robin Thomas, Reducibility in the Four-Color Theorem, 24 January 2014, ArXiV ID: 1401.6481

[14] Arthur Benjamin , Gary Chartrand, Ping Zhang, The Fascinating World of Graph Theory (Coloring Graphs), Princeton University Press, June 06 2017, DOI: 10.23943/princeton/9780691175638.003.0011 